\title{
Correction to: Pattern Recognition and Artificial Intelligence
}

Chawki Djeddi (D), Yousri Kessentini (D), Imran Siddiqi, and Mohamed Jmaiel

\section{Correction to: \\ C. Djeddi et al. (Eds.): Pattern Recognition and Artificial Intelligence, CCIS 1322, https://doi.org/10.1007/978-3-030-71804-6}

The affiliations of the first two volume editors were incorrect. They were corrected to:

Chawki Djeddi

Larbi Tebessi University

Tebessa, Algeria

Yousri Kessentini

Digital Research Center of Sfax

Sfax, Tunisia 